\title{
Toxic excipients in medications for neonates in Brazil
}

\author{
Alcidésio Souza Jr • Djanilson Santos • Said Fonseca • \\ Marina Medeiros • Lívia Batista • Mark Turner • \\ Helena Coelho
}

Received: 14 November 2013 /Revised: 8 January 2014 / Accepted: 22 January 2014 / Published online: 6 February 2014

(C) Springer-Verlag Berlin Heidelberg 2014

\begin{abstract}
The aim was to describe the exposure to excipients among neonates hospitalised in the neonatal intensive care unit (NICU) of a public hospital in Brasilia, Brazil. This was a retrospective study based on medicines that were prescribed electronically to neonates ( $\leq 28$ days) who were admitted to the NICU of a hospital in Brasilia between January 1 and March 31, 2012. Excipients were identified from the medicine package leaflets and were classified according to toxicity. Seventy-nine infants received a total of 1,303 prescriptions comprising 77 formulations and 70 active drugs. Eighty-six excipients were identified, of which, 9 were harmful excipients (HE) and 48 were potentially harmful excipients (PHE). Almost all the neonates $(98.7 \%)$ were exposed to at least one HE and PHE. Preterm neonates ( $n=64 ; 1,502$ neonate days) presented high risk of exposure to polysorbate $80(3.26 / 100$ neonate days), sodium hydroxide (3.39), PG (3.19) and propylparaben (3.06). Full-term neonates $(n=15 ; 289$ neonate
\end{abstract}

Communicated by Patrick Van Reempts

A. Souza Jr

Mother and Child Hospital of Brasilia, SGAS, Av. L2 Sul, Quadra

608, Módulo A Asa Sul, Brasília, Federal District, Brazil

A. Souza Jr $(\bowtie) \cdot$ S. Fonseca $\cdot$ H. Coelho

Doctoral Program on Development and Technological Innovation in

Drugs, Federal University of Ceará, Fortaleza, Ceará, Brazil

e-mail: alcidesiojr@gmail.com

D. Santos $\cdot$ M. Medeiros $\cdot$ L. Batista $\cdot$ H. Coelho

Postgraduate Programme on Pharmaceutical Sciences, Federal

University of Ceará, Fortaleza, Ceará, Brazil

D. Santos

Federal University of Recôncavo da Bahia, Santo Antônio de Jesus, Bahia, Brazil

M. Turner

Department of Women's and Children's Health, University of Liverpool, Liverpool, UK days) presented risks in relation to phenol (4.84), ethanol (3.8) and sodium citrate (3.46). Conclusion: Neonates in NICUs in Brazil are exposed to a wide variety of HE and PHE with unpredictable results. Safer alternatives are needed, as well as further studies on the subject.

Keywords Harmful excipients · Potentially harmful excipients $\cdot$ Neonate $\cdot$ Drug

\section{Introduction}

It is estimated that critically ill newborns in neonatal intensive care units (NICUs) receive up to15 to 20 medicines routed intravenously $[10,15]$ and may be exposed to over 20 different excipients per day, depending on the number and dosage form of the drugs that they receive during hospitalisation [34]. This merits great concern, given the limited knowledge available regarding the full impact of developmental immaturity on the safety of drugs and their excipients in newborns, especially in those that are more immature and/or affected by diseases and specific conditions [1, 13, 14].

Serious and even life-threatening adverse events have been associated with exposure to the excipients present in drugs, when administered in higher doses or to vulnerable population groups such as neonates, particularly, those with low birth weight. These reactions may occur because of the immaturity of the organs responsible for biotransformation and elimination, which results in accumulation of these substances and their ensuing the toxicity, as has been observed in relation to propylene glycol (PG) [2, 13, 14, 26, 34].

Currently, certain excipients are recognised as toxic to newborns if they are given at a high dose. These include sodium benzoate, PG, parabens (methyl and propyl parahydroxybenzoate), sodium saccharin, benzyl alcohol (BA), benzalkonium chloride, polysorbate 80 and ethanol, 
which are present in medicines used commonly in this population worldwide [17, 21, 26, 28, 32-34]. Lass et al. [17] identified exposure of hospitalised neonates in Estonia to these and other excipients and made an attempt to categorise these substances in terms of safety, according to published information.

We are not aware of any data relating to excipient exposure among neonates in countries that are not included in the region covered by the International Conference on Harmonisation of Technical Requirements for Registration of Pharmaceuticals for Human Use (ICH), such as Brazil. This paper describes the exposure to excipients among neonates admitted to the NICU of a public care service in Brasilia, Brazil, applying the categories of Lass et al. [17] for risk analysis.

\section{Methods}

This was a retrospective study based on data from prescribed formulations, which was conducted in the NICU of a mother and child hospital in Brasilia, Federal District, Brazil, between January and March 2012. This is a tertiary-level institution specialising in caring for pregnant women and children, with 345 inpatient beds, of which, 46 beds are in the NICU (26 active currently) and 14 are in the paediatric surgery sector. The study subjects were neonates (postnatal age $\leq 28$ days) who were admitted for more than $24 \mathrm{~h}$ during the study period. Data regarding intravenous hydration, vaccines, blood and parenteral nutrition were not recorded. Patients with incomplete clinical data, incomplete prescription or prescription of intravenous hydration alone were excluded. The research was approved by the Research Ethics Committees of the Health Department of the Federal District (Brazil), under Protocol No. 021/2012.

Data gathering

The prescription data and other information were gathered from the patients' electronic medical records. The following data were recorded on specific forms: gestational age, birth weight, gender, date of birth, diagnoses, prescribed formulations data (name of the drug, laboratory, dosage form, administration beginning and end of treatment) and outcome within the observation period (discharge, death, transfer or remained hospitalised).

Neonates were classified according to the gestational age (GA) as preterm $(<37$ weeks of GA) and full-term ( $\geq 37$ weeks); and according to gestational weight as very low weight $(<1,500 \mathrm{~g})$, low birth weight $(1,500$ to $2,500 \mathrm{~g})$ and above $(>2,500 \mathrm{~g})[10]$.

Drugs were grouped into therapeutic classes in accordance with the Anatomical Therapeutic Chemical classification [35].
The composition of prescription formulations were determined from the drug package leaflets. If this information was not found, the electronic package leaflet database of the National Agency for Sanitary Surveillance (ANVISA) [3] and the website http://www.bulasmed.com.br [6] were consulted.

The excipients identified were categorised as proposed by Lass et al. [17], into four categories: (a) Potentially harmful and known to be harmful-adverse reactions reported; (b) Potentially safe - no adverse reactions reported; (c) No safety data found - no data found in the literature on human exposure and toxicity; (d) Manufacturer's description of the excipients does not allow a specific literature search. The excipients for which no classification was included in the paper by Lass et al. [17] were categorised based on the information present in the Handbook of Pharmaceutical Excipients (6th edition) [24] and on the websites http://hazmap.nlm.nih.gov [31] and http://toxnet.nlm.nih.gov [32] (Fig. 1). The excipient classification was certified independently by three evaluators (MSGM, LAAB and ASSJ) and cases of discordance were resolved through discussion among them to reach a consensus.

\section{Data processing and statistical analysis}

The exposure was estimated using the number of medications containing an excipient divided by the total number of prescriptions and using the incidence rate (IR) for each excipient. In IR, the numerator was the number of neonates exposed to each excipient once or more times and the denominator was the total number of neonate days at risk of using an excipient. The IR made it possible to evaluate the exposure of newborn infants to each excipient. For analysis, the IR was subdivided according to the GA. The data were stored in Excel for Windows version 7 and analysed in the Statistical Package for the Social Sciences version 18 , using simple descriptive analysis.

\section{Results}

Characteristics of the neonates

During the study period, 89 patients were admitted to the NICU, among whom 10 (11.2\%) were excluded (4 had postnatal ages of more than 28 days of postnatal age, 4 presented incomplete data, and 2 only received intravenous hydration or parenteral nutrition). Of the 79 neonates included in the study, $64(81.0 \%)$ were preterm and $15(19.0 \%)$ were full-term; the median GA was 33.4 weeks (GA range 2240 weeks); 40 (50.6\%) were male; the mean length was $42.1 \mathrm{~cm}$ (standard deviation $(\mathrm{SD}) \pm 5.3 \mathrm{~cm}$ ); the mean birth weight was $1,793.0 \mathrm{~g}(\mathrm{SD} \pm 855.7 \mathrm{~g})$; and $63(80.7 \%)$ had low birth weight $(<2,500 \mathrm{~g})$, including 35 of very low birth weight 
Fig. 1 Categorisation procedure for excipients found in prescription drugs in the NICU

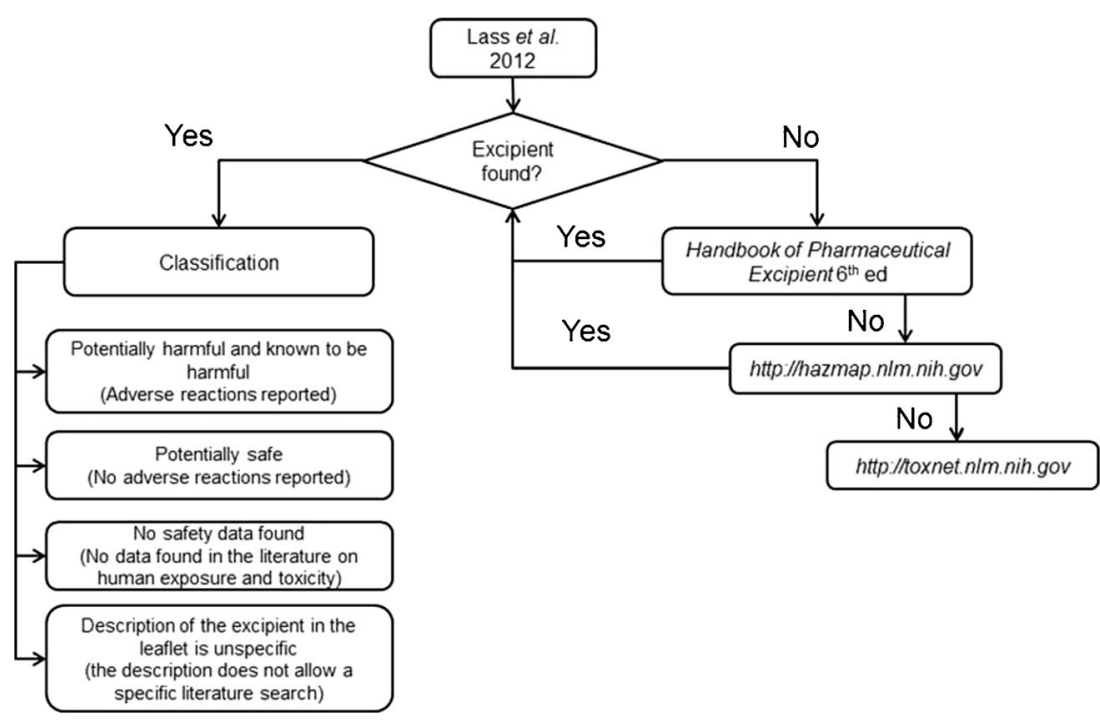

$(<1,500 \mathrm{~g})$. The median length of hospital stay was 18 days (range: $1-75$ days; mean 22.6 days; $\mathrm{SD} \pm 17.7$ days), thus totalling to 1,791 neonate days, and 24 deaths occurred during the period. The most frequent diagnoses that led to admission to the NICU were: respiratory distress $(30.1 \%)$, jaundice $(11.6 \%)$, sepsis $(8.4 \%)$, bacterial/fungal infection (6.8\%) and hyaline membrane disease $(5.3 \%)$.

\section{Prescribed formulations}

The neonates in the study received 1,303 prescriptions (median: 10.0 prescription/patient; interquartile range (IQR) 8.5; range 1-70) for a total of 733 prescribed formulations (median nine formulations/patient; interquartile range 4.5; range 1-26) with 77 different formulations and 70 different active pharmaceutical ingredients (API). The preterm neonates had a higher number of prescriptions (median 11; IQR 22; range 1-70 prescription/neonate); for the full-term neonates, the median number was 9 (IQR 7; range 1-19 prescription/ neonate). The therapeutic classes that were most prescribed were drugs for the alimentary tract and metabolism $(n=21$; $29.2 \%)$, anti-infective for systemic use $(n=16 ; 22.2 \%)$ and drugs for the cardiovascular system $(n=11 ; 13.9 \%)$. The five formulations that were most prescribed were aminophylline as an injection solution $(n=566 ; 9.8 \%)$, multivitamin without minerals as an injectable solution $(n=409 ; 7.0 \%)$, multivitamin without minerals as an oral solution $(n=404 ; 6.9 \%)$, domperidone oral solution $(n=324 ; 5.6 \%)$ and ranitidine as an injectable solution ( $n=294 ; 5.0 \%)$.

Regarding the dosage form of the 77 formulations, $51.9 \%$ (40) were injectable, $22.0 \%$ oral solutions, $9.0 \%$ tablets, $7.8 \%$ oral suspensions, $2.6 \%$ oral emulsions, $2.6 \%$ ophthalmic solutions, $1.3 \%$ pulmonary inhalation solution, $1.3 \%$ sterile suspension for intratracheal administration and $1.3 \%$ ointments.
Classification of the excipients present in prescribed formulations

Of the 77 formulations investigated, it was only possible to obtain the drug package leaflet of 58 and only 53 of these cited the excipients. The mean number of excipients per formulation was 5.3 (range 1-14) with a total of 86 excipients, of which, 21 were mentioned in the paper by Lass et al. [17]. The excipients were categorised as follows: (a) potentially harmful and known to be harmful $(n=57 ; 66.2 \%)$; (b) potentially safe $(n=8$; $9.3 \%)$; (c) no safety data found ( $n=1 ; 1.2 \%)$ and (d) description of the excipients in the leaflet is unspecific $(n=20 ; 23.3 \%)$. Excipients in category "a" were subdivided into harmful excipients (HE, $n=9$ ) and potentially harmful excipients (PHE, $n=48$ ) which were found in 48 formulations, among which 4 formulations only presented HE, 24 only presented PHE and 20 had both types of excipients (Tables 1 and 2). The excipients citric acid, hydrochloric acid, stearic acid, water, starch, microcrystalline cellulose, sodium chloride and simethicone were included in category "b" and were present in 43 formulations, used as diluents, suspending agents, solvents and antioxidants. Category "c" included carmellose sodium present in two formulations and functioned as suspending agent. Category "d" included flavouring agents, scents, and colourants, such as mint essence, artificial orange flavour and iron oxide yellow colourant.

Neonates' exposure to harmful and potentially harmful excipients

Sixty-nine neonates $(87.3 \%)$ were exposed to one or more excipients known to be harmful (HE), with median of three HE per neonate (IQR 8; range: 1-8) and a median of three formulations with HE per neonate (IQR 8; range: 1-9). Almost all the preterm neonates were exposed to HE 


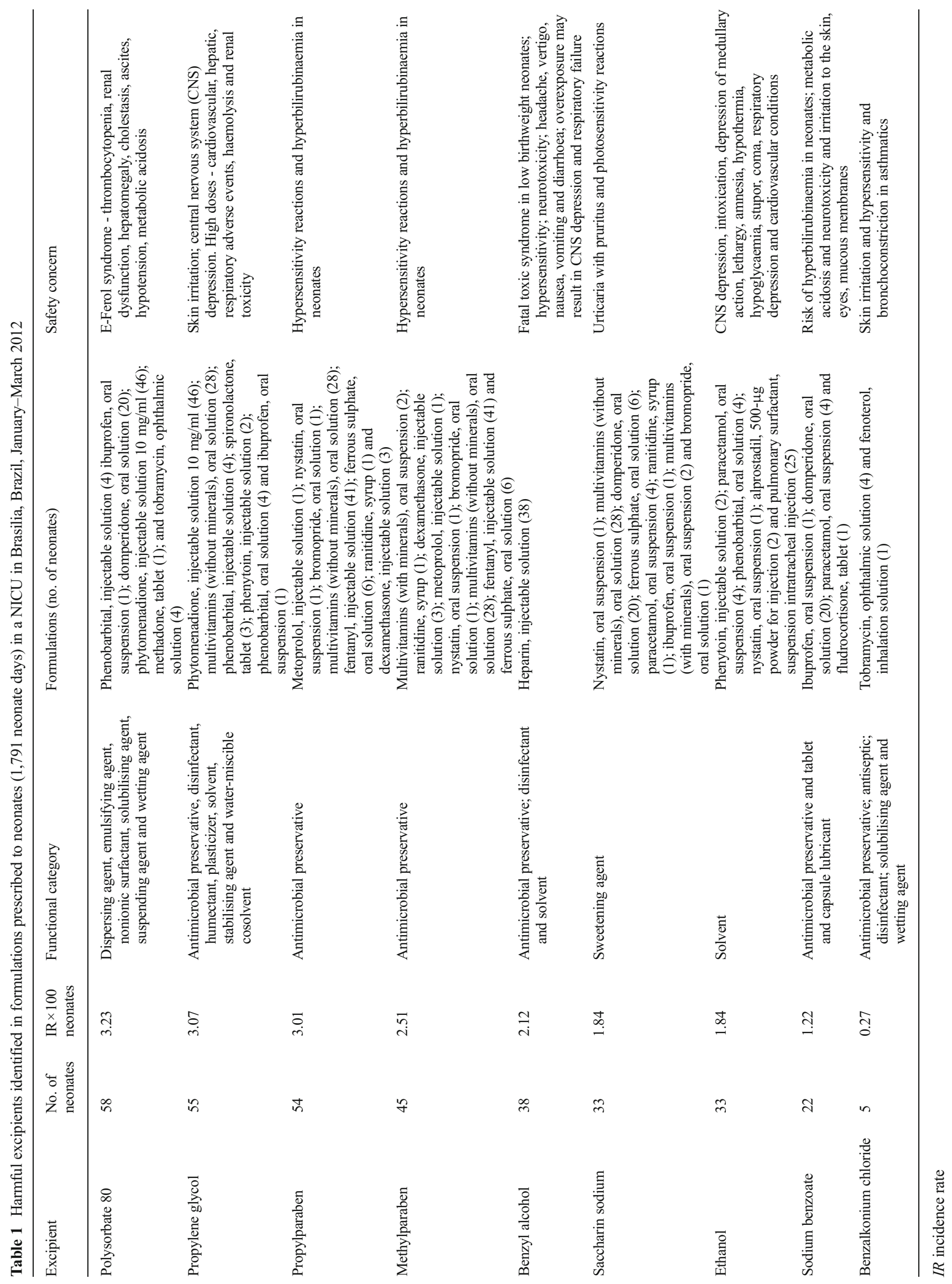




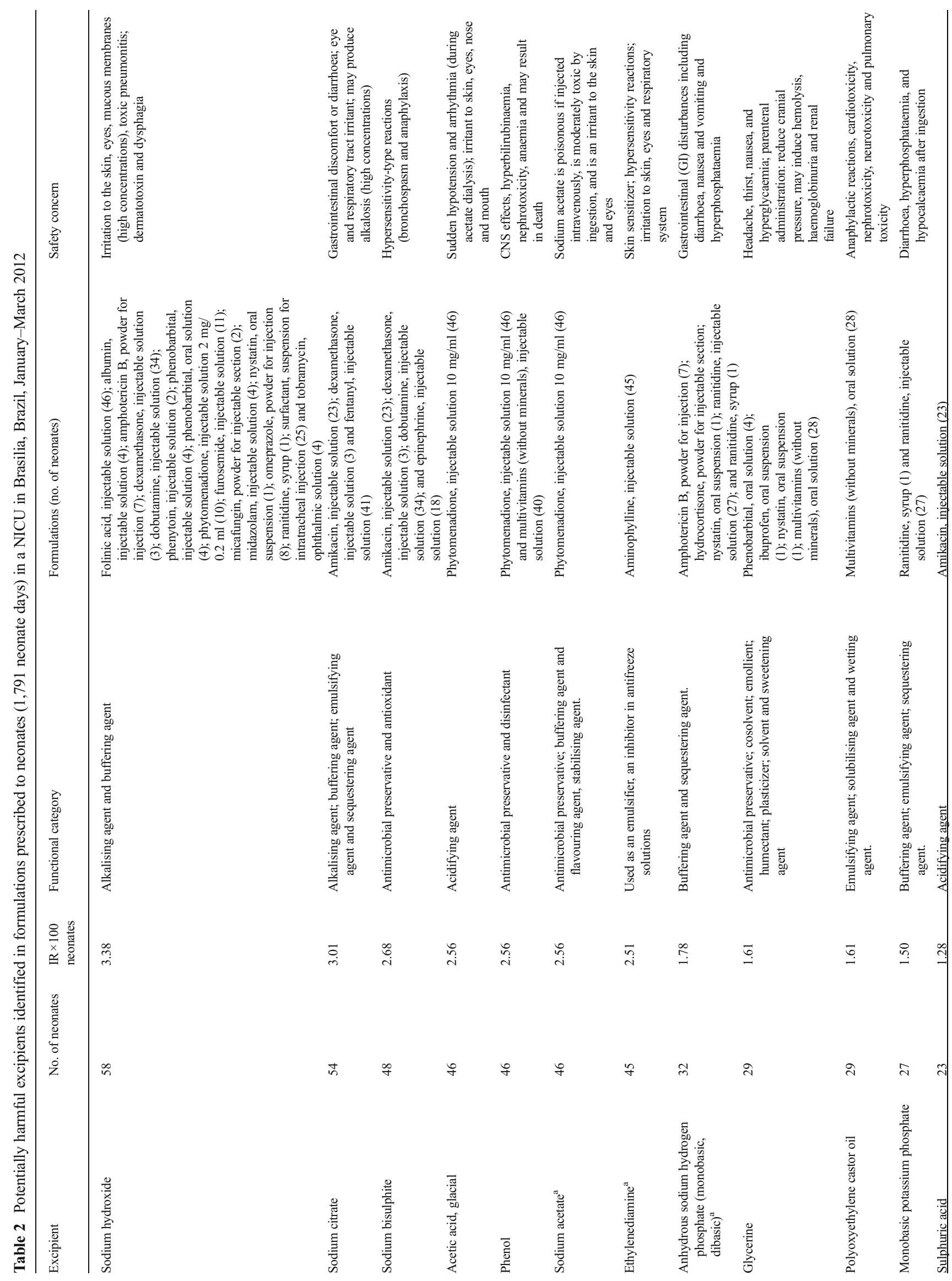




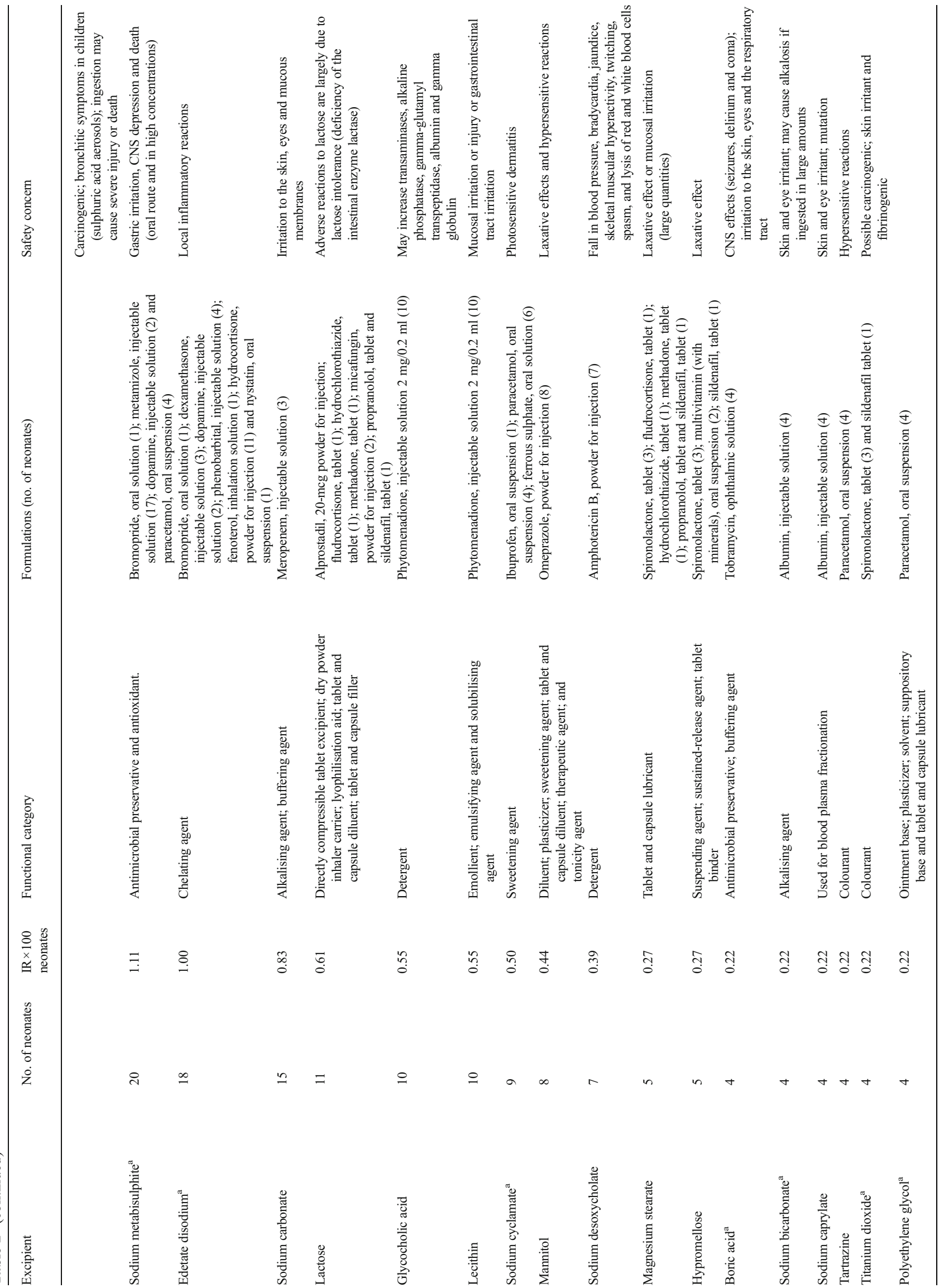




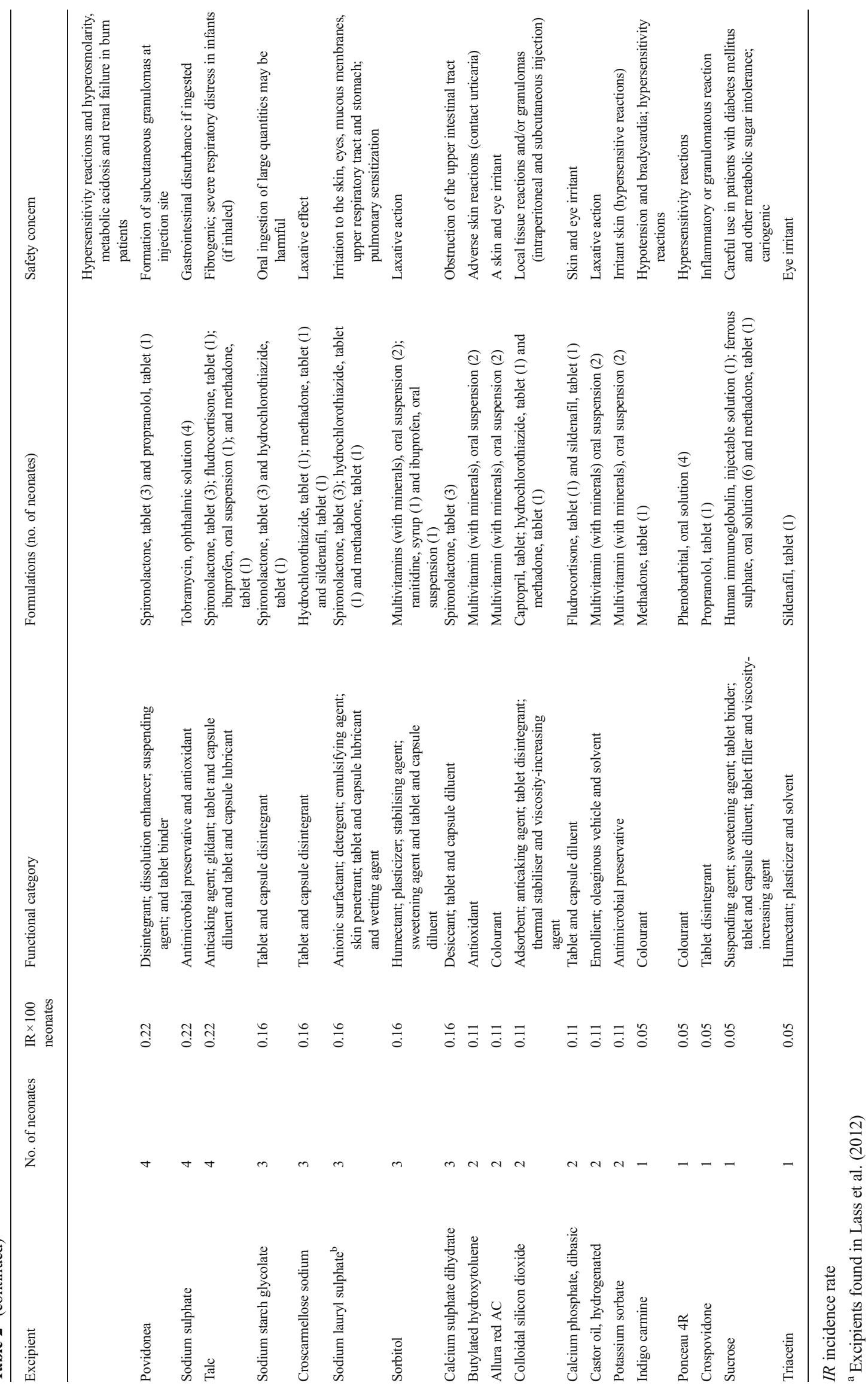


$(87.5 \%)$ and $86.6 \%$ of full-term neonates were exposed at least once. Among the formulations that were prescribed with HE, preterm neonates presented a median number of three formulations with HE (IQR 2; range: 1-9), and for the fullterm neonates, the median was also three formulations with HE (IQR 2.5; range: 1-9).

Table 1 shows the IR of excipients per 1,791 neonate days during the study period. Polysorbate 80 had an IR of 3.23 per 100 neonate days, meaning that for every 100 neonates who remained hospitalised for 1 day, three were exposed to this excipient. The risk of exposure to other excipients was less than this, in the following decreasing order: $P G$, propylparaben, methylparaben, BA, saccharin, ethanol, sodium benzoate and benzalkonium chloride.

According to the IR and GA, the preterm neonates $(1,502$ neonate days) were at increased risk of exposure to polysorbate 80 (3.26/100 neonate days), followed by PG (3.19), propylparaben (3.06), methylparaben (2.39), ethanol (2.33), BA (2.06), sodium saccharin (1.86), sodium benzoate (1.19) and benzalkonium chloride (0.33). The full-term neonates (289 neonate days) presented greater risk in relation to ethanol (3.8), methylparaben (3.11), polysorbate 80 (3.11), propylparaben (2.76), BA (2.42), PG (2.42), sodium saccharin (1.73) and sodium benzoate (1.38). The full-term neonates were not exposed to benzalkonium chloride.

Nearly all the newborns (98.7\%) were exposed to one or more PHE. Among the formulations prescribed, the median number of PHE included in prescriptions for the neonates was 7 (IQR 6.2; range 1-19). Among these, the median for preterm neonates was 7 (IQR 6.0; range 1-19) and for full-term neonates were 2.5 (IQR 6.0; range 1-7). The risk of exposure was higher for sodium hydroxide, sodium citrate, sodium bisulphite, acetic acid and phenol (Table 2).

In relation to IR and PHE, the preterm neonates (1,502 neonate days) presented increased risk of exposure to sodium hydroxide (3.39/100 neonate days), ethylenediamine (2.99), sodium citrate (2.92), acetic acid (2.92) and sodium acetate (2.92). The full-term neonates (289 neonate days) presented increased risk of exposure to phenol (4.84/100 neonate day), sodium citrate (3.46), anhydrous sodium hydrogen phosphate (3.11), sodium hydroxide (2.42) and sodium bisulphite (2.42).

\section{Discussion}

To our knowledge, this is the first report on excipient exposure from a country that is not included in a region represented in the $\mathrm{ICH}$ and the first to express the rate of excipient exposure according to neonate days. The present study describes the exposure of an unselected group of neonates that were hospitalised in a NICU in Brasilia, the capital of Brazil, to a wide variety of $\mathrm{HE}$ or PHE that are present in commonly used medicines. This is a situation similar to that found in other countries [17, 21, 26, 33, 34]. The study population consisted primarily of preterm neonates, which increases the significance of these observations, given the greater vulnerability of this population to the toxicity of drugs and excipients, and the little that is known about this subject $[1,13]$.

Prescribing in public institutions in Brazil is standardised at each institution based on the list of essential medicines in the country, with variations in the profile with regard to local factors, product availability and prices [7]. The registration of new drugs is regulated by ANVISA, yet there are no specific rules for paediatric medicines or restrictions on the presence of excipients in them.

The therapeutic classes that were found to be most prescribed in this study were drugs for the alimentary tract and metabolism, anti-infective drugs for systemic use and drugs for the cardiovascular system, which are commonly used in NICUs [5, 9, 22]. We identified a large number of excipients with different functions and safety profiles $[12,17,23,33$, 34]. About two-thirds of the excipients identified were classified as $\mathrm{HE}$ and PHE, which were present in formulations that were used frequently and concurrently. This highlights the risk of addition, accumulation and enhancement of toxic effects, including interaction with the API present in the formulations [12, 19, 26-28].

Nearly all the neonates were exposed to HE and PHE that whose toxicity appears to be higher at lower gestational age [2, 17, 26, 34]. The number of formulations containing HE and PHE were similar to what was found by Lass et al. [17], differing only in the comparison between groups of GA in relation to $\mathrm{HE}$. The neonates of the present study were at increased risk of exposure to polysorbate 80 ( 7 formulations/ 610 prescriptions), thus differing from the findings of Lass et al. [17] (4 formulations/70 prescriptions). This was despite the limited overlap in excipients between the two surveys ( $24 \%$ of excipients seen in Brazil were reported in Estonia). The European Study of Neonatal Exposure to Excipients preliminary results of the point prevalence survey (ESNEEPPS) reported that $42 \%$ of 825 neonates were exposed to parabens [21]. These differences may have occurred because we have included all the formulations that were prescribed (e.g., phytomenadione injections). This product contains polysorbate, an excipient that is associated with E-Ferol syndrome which occurs in neonates that receive vitamin E preparations with large amounts of polysorbate 80 [24].

With regard to exposure to alcohols, the neonates in our study had a higher risk of being exposed to PG, BA and ethanol, differently from Lass et al. [17] who demonstrated lower exposure to BA, PG and ethanol. However, our findings were similar to the preliminary ESNEE-PPS results, if benzoic acid is considered to be a BA metabolite [21]. The acceptable daily intakes of PG and ethanol are not well known for this population, but their use is not recommended for children under 4 years of age [11]. The pharmacokinetics of PG have 
been described, but the "safe" circulating concentration remains uncertain [8]. Furthermore, recent data suggest that there is a lower limit of PG tolerance in neonates [16]; for example, no adverse reactions were associated with short-term exposure to a median PG dose of $34 \mathrm{mg} / \mathrm{kg} /$ day [2]. It has been acknowledged that large amounts of PG and its interaction with ethanol, as reported by the United States Food and Drug Administration in the case of lopinavir/ritonavir $\left(\right.$ Kaletra $\left.^{\mathbb{R}}\right)$ [30], possibly contributes towards the toxicity of PG. BA (which is contraindicated by the FDA and the European Medicines Agency in formulations for neonates) is present in heparin sodium injection solution, which is usually used to extend the access of an umbilical arterial catheter in neonates. This was also used in Estonia according to Lass et al. [17]. In a study conducted in the USA, Shebab et al.[26] observed that neonates received daily intake of BA and PG 21 to 180 that was times higher than the maximum recommended daily dose for adults ( 5 and $25 \mathrm{mg} / \mathrm{kg} /$ day, respectively). Also, Whittaker et al. [34] in England observed exposure to significant daily intake of ethanol among neonates who weighed between 1.0 and $3.5 \mathrm{~kg}$ ( 0.2 to $1.8 \mathrm{~mL} /$ week, not adjusted by weight).

The toxicity among neonates relating to the preservative sodium benzoate is assumed to derive from benzoic acid accumulation, which may cause metabolic acidosis and neurotoxicity [20]. We observed that sodium benzoate was present in four of the prescribed formulations, among which domperidone oral solution is frequently used in the treatment of gastrointestinal reflux, although its use is controversial [18]. Lass et al. [17] reported that benzoic acid was present in four formulations, among which simethicone oral suspension was the one most prescribed.

Propyl and methylparaben were present in eight and nine formulations, respectively: injectable fentanyl solution (251 prescriptions) and multivitamins without minerals in oral solution (409 prescriptions) were the most frequently used of these formulations. These excipients are widely used as preservatives in the pharmaceutical and food industries, and are often encountered in medications used in NICUs, as suggested by some authors [4, 17, 21]. Lass et al. [17] also reported that there was a high frequency of prescribed formulations containing propyl and methylparaben, with gentamicin injection as the type most prescribed (200/1,971 prescriptions). Also, the preliminary ESNEE results [21] showed that there was high prevalence of exposure to parabens ( $42 \%$ out of 825 neonates in 21 countries). In research on paediatric formulations marketed in the Netherlands (3,542 formulations), van Riet-Nales et al. [33] detected the presence of methyl and propylparaben in oral liquid formulations (77 and 45, respectively) and injectable formulations ( 9 and 1 , respectively). Our results may differ from those of Lass et al. [17] and from the preliminary ESNEE results [21] for a number of reasons, including the way in which parabens were recorded.
However, even considering the frequency of parabens as a group, the polysorbate exposure appears to be greater in Brazil than in Europe.

Sodium cyclamate, sorbitol and sodium saccharin were identified. The last of these was present in the most widely prescribed products (33 neonates/909 prescriptions), i.e., multivitamins without minerals in oral solution (40 neonates/404 prescriptions) and domperidone oral solution (20 neonates/ 324 prescriptions). It was also found by Lass et al. [17] in six formulations (173/1,971 prescriptions), among which simethicone oral suspension stood out (108 prescriptions). The use of sweetening agents in formulations for neonates is not recommended because of the lack of established safety data and the known risks of hypersensitivity (sodium saccharin) and laxative action (sorbitol) [12, 24]. However, it also needs to be taken into consideration that the production of excipient-free formulations may not be economically viable.

The clinical significance of these results is unclear, mainly because of the lack of quantitative information about excipients in medicines in our setting. This problem has been reported by other authors [2, 17, 25, 33, 34]. Manufacturers regard this information as proprietary and commercially sensitive. This makes it difficult to assess the extent of exposure to excipients, which was also reported by the ESNEE [25] and the PG experience [16]. The lack of quantitative information about excipients in medicines hinders research and prevents rational risk management by clinicians and regulators [29]. The present study was conducted over a short period and it should be borne in mind that exposure to excipients may continue during and after hospitalisation, as demonstrated by Whittaker et al. [34]. Moreover, it should be noted that the reports of excipient toxicity in neonates reflect unusually high exposures. Routine surveillance for the known adverse effects of excipients among neonates has not been reported often, although no problems were revealed as effects of PG [2]. Excipients are sometimes required to solubilise the medicine or to preserve the formulation, so as to extend the shelf-life of the medicines and improve the marketability of these medicines.

This paper provides the first detailed description of neonate exposure to toxic excipients in a NICU in Brazil and calls their risks into question. Our study applies the classification proposed by Lass et al. [17] to the reported excipients and complements that report with the inclusion of excipients not mentioned by these authors. There is clearly a significant difference between excipient exposure in different countries which needs to be accounted for in regulatory action and research. These differences may reflect differences in choice of active agents, differences in availability (due to market availability or price) or regulatory differences.

We recognise that this study has limitations that were inherent to its retrospective nature and to the fact that it was based on records from a single NICU. On the other hand, the 
electronic medical records with the analysis of over 1,303 prescriptions accumulated during 3 months, gives more reliability to the data. These results can be generalised to neonates in other public NICUs in Brazil, although there are regional and local differences in terms of the characteristics of these populations and health service practices.

It is important to remind prescribers about the need to take into account the excipients present in the medicines prescribed for neonates. Monitoring for adverse events potentially related to excipients should be part of the drug use routine in the NICU, as proposed by Giacoia et al. [13]. Neonates have not yet benefited appreciably from international efforts to promote the development of better medicines for children. Therapeutic arsenal for this population group remains very limited and the risk-benefit balance of excipients is not adequately addressed in many situations. However, promising studies are being conducted in Europe, like the European Study of Neonatal Excipient Exposure, which aim to assess and mitigate the risk posed by excipients in neonates [28]. Despite the risks, it is important to remember that excipients are necessary in medicines and it may not be economically feasible to develop medicines that do not contain excipients that are targeted exclusively to neonates. The risks can be minimised by actions such as excluding excipients associated with harm whenever possible, limiting the concentrations of excipients which are indispensable and informing clinicians about the quantitative composition of medicines. These actions should be regulatory requirements and good practice in the pharmaceutical industry in all countries, including Brazil.

Acknowledgement This study was supported by grants from the Institute of Health Science - Research and Teaching Foundation-FEPECS.

Conflict of interest The authors declare that they have no personal financial relationship with the sponsoring organisations or any other potential conflicts of interest.

\section{References}

1. Allegaert K, Langhendries JP, van den Anker J (2013) Do we need neonatal clinical pharmacologists? Eur J Pediatr 172(4):429-435. doi:10.1007/s00431-012-1734-4

2. Allegaert K, Vanhaesebrouck S, Kulo A, Cosaert K, Verbesselt R, Debeer A, de Hoon J (2010) Prospective assessment of short-term propylene glycol tolerance in neonates. Arch Dis Child 95(12):10541058. doi:10.1136/adc. 2010.190330

3. ANVISA (2013) Bulário Eletrônico. http://www4.anvisa.gov.br/ BularioEletronico. Accessed 01 June 2013

4. Calafat AM, Weuve J, Ye X, Jia LT, Hu H, Ringer S, Huttner K, Hauser R (2009) Exposure to bisphenol A and other phenols in neonatal intensive care unit premature infants. Environ Heal Perspect 117(4):639-644. doi:10.1289/ehp.0800265

5. Carvalho CG, Ribeiro MR, Bonilha MM, Fernandes M Jr, Procianoy RS, Silveira RC (2012) Use of off-label and unlicensed drugs in the neonatal intensive care unit and its association with severity scores. $\mathrm{J}$ Pediatr (Rio J) 88(6):465-470. doi:10.2223/JPED.2231

6. Centralx (2013) Sistemas Inteligentes em Saúde. http://www.bulas. med.br. Accessed 01 June 2013

7. Coelho HL, Rey LC, Medeiros MS, Barbosa RA, Cruz Fonseca SG, Costa PQ (2013) A critical comparison between the World Health Organization list of essential medicines for children and the Brazilian list of essential medicines (Rename). J Pediatr (Rio J) 89(2):171-178. doi:10.1016/j.jped.2013.03.004

8. De Cock RF, Knibbe CA, Kulo A, de Hoon J, Verbesselt R, Danhof M, Allegaert K (2013) Developmental pharmacokinetics of propylene glycol in preterm and term neonates. Br J Clin Pharmacol 75(1): 162-171. doi:10.1111/j.1365-2125.2012.04312.x

9. Dell'Aera M, Gasbarro AR, Padovano M, Laforgia N, Capodiferro D, Solarino B, Quaranta R, Dell'Erba AS (2007) Unlicensed and offlabel use of medicines at a neonatology clinic in Italy. Pharm World Sci: PWS 29(4):361-367. doi:10.1007/s11096-006-9081-z

10. EMEA/536810/2008 (2010) Guideline on the investigation of medicinal products in the term and preterm neonate. EMEA. http://www.ema.europa.eu/docs/en_GB/document_library/ Scientific_guideline/2009/09/WC500003750.pdf. Accessed 01 February 2013

11. EMEA/CHMP/PEG/194810/2005 (2006) Reflection paper: formulations of choice for the paediatric population. EMEA. http://www. ema.europa.eu/docs/en_GB/document_library/Scientific_guideline/ 2009/09/WC500003782.pdf. Accessed 01 February 2013

12. Fabiano V, Mameli C, Zuccotti GV (2011) Paediatric pharmacology: remember the excipients. Pharmacol Res: Off J Ital Pharmacol Soc 63(5):362-365. doi:10.1016/j.phrs.2011.01.006

13. Giacoia GP, Taylor-Zapata P, Zajicek A (2012) Drug studies in newborns: a therapeutic imperative. Clin Perinatol 39(1):11-23. doi:10.1016/j.clp.2011.12.016

14. Jacqz-Aigrain E (2011) Drug policy in Europe research and funding in neonates: current challenges, future perspectives, new opportunities. Early Hum Dev 87(Suppl 1):S27-S30. doi:10.1016/j. earlhumdev.2011.01.007

15. Kalikstad B, Skjerdal A, Hansen TW (2010) Compatibility of drug infusions in the NICU. Arch Dis Child 95(9):745-748. doi:10.1136/ adc. 2009.174268

16. Kulo A, de Hoon JN, Allegaert K (2012) The propylene glycol research project to illustrate the feasibility and difficulties to study toxicokinetics in neonates. Int J Pharm 435(2):112-114. doi:10.1016/ j.ijpharm.2012.05.014

17. Lass J, Naelapaa K, Shah U, Kaar R, Varendi H, Turner MA, Lutsar I (2012) Hospitalised neonates in Estonia commonly receive potentially harmful excipients. BMC Pediatr 12:136. doi:10.1186/1471-2431$12-136$

18. Malcolm WF, Cotten CM (2012) Metoclopramide, H2 blockers, and proton pump inhibitors: pharmacotherapy for gastroesophageal reflux in neonates. Clin Perinatol 39(1):99-109. doi:10.1016/j.clp. 2011.12.015

19. Nahata MC (2009) Safety of "inert" additives or excipients in paediatric medicines. Arch Dis Child Fetal Neonatal Ed 94(6):F392-F393. doi:10.1136/adc.2009.160192

20. Nair B (2001) Final report on the safety assessment of benzyl alcohol, benzoic acid, and sodium benzoate. Int J Toxicol 20(Suppl 3):23-50

21. Nellis G, Metsvaht T, Lass J, Varendi H, Turner MA, Lutsar I (2013) European Study of Neonatal Excipient Exposure (ESNEE): preliminary results of the point prevalence survey. In: 14th Biannual Congress of the European Society for Developmental Perinatal and Pediatric Pharmacology (EDPPP), Salzburg, Austria, German Medicam Science GMS Publishing House, Düsseldorf. http://www. esdppp.org/site/wp-content/uploads/2013/06/ESDP\%20ibook\% 20final\%201.1\%20light.pdf. Accessed 04 December 2013

22. Neubert A, Lukas K, Leis T, Dormann H, Brune K, Rascher W (2010) Drug utilisation on a preterm and neonatal intensive care unit 
in Germany: a prospective, cohort-based analysis. Eur J Clin Pharmacol 66(1):87-95. doi:10.1007/s00228-009-0722-8

23. Pifferi G, Restani P (2003) The safety of pharmaceutical excipients. Farmaco 58(8):541-550

24. Rowe RC, Sheskey PJ, Quinn ME (2009) Handbook of pharmaceutical excipients, 6th edn. Pharmaceutical Press, London

25. Salunke S, Brandys B, Giacoia G, Tuleu C (2013) The STEP (Safety and Toxicity of Excipients for Paediatrics) database: part 2 - the pilot version. Int J Pharm 457(1):310-322. doi:10.1016/j.ijpharm.2013. 09.013

26. Shehab N, Lewis CL, Streetman DD, Donn SM (2009) Exposure to the pharmaceutical excipients benzyl alcohol and propylene glycol among critically ill neonates. Pediatr Crit Care Med: J Soc Crit Care Med World Fed Pediatr Intensive Crit Care Soc 10(2):256-259. doi: 10.1097/PCC.0b013e31819a383c

27. Tuleu C, Breitkreutz J (2013) Educational paper: formulation-related issues in pediatric clinical pharmacology. Eur J Pediatr 172(6):717720. doi:10.1007/s00431-012-1872-8

28. Turner MA, Duncan J, Shah U, Metsvaht T, Varendi H, Nellis G, Lutsar I, Vaconsin P, Storme T, Rieutord A, Nunn AJ (2013) European study of neonatal exposure to excipients: an update. Int $\mathbf{J}$ Pharm 457(1):357-358. doi:10.1016/j.jpharm.2013.08.078

29. Turner MA, Duncan JC, Shah U, Metsvaht T, Varendi H, Nellis G, Lutsar I, Yakkundi S, McElnay JC, Pandya H,
Mulla H, Vaconsin P, Storme T, Rieutord A, Nunn AJ (2013) Risk assessment of neonatal excipient exposure: lessons from food safety and other areas. Adv Drug Deliv Rev. doi:10.1016/j.addr.2013.11.003

30. US/FDA (2011) FDA drug safety communication: serious health problems seen in premature babies given Kaletra (lopinavir/ritonavir) oral solution. http://www.fda.gov/Drugs/DrugSafety/ucm246002. $\mathrm{htm}$. Accessed 05/12/2013

31. US/NLM (2013) Haz-Map ${ }^{\circledR}$. U.S. National Library of Medicine. http://hazmap.nlm.nih.gov. Accessed 10 July 2013

32. US/NLM/SIS (2013) TOXNET. U.S. National Institutes of Health. http://toxnet.nlm.nih.gov/. 10 July 2013

33. van Riet-Nales DA, de Jager KE, Schobben AF, Egberts TC, Rademaker CM (2011) The availability and age-appropriateness of medicines authorized for children in the Netherlands. $\mathrm{Br} \mathrm{J}$ Clin Pharmacol 72(3):465-473. doi:10.1111/j.1365-2125.2011. 03982.x

34. Whittaker A, Currie AE, Turner MA, Field DJ, Mulla H, Pandya HC (2009) Toxic additives in medication for preterm infants. Arch Dis Child Fetal Neonatal Ed 94(4):F236-F240. doi:10.1136/adc.2008. 146035

35. WHO (2010) Anatomical-therapeutical-chemical (atc) classification-index and guidelines. http://www.who.int/classifications/ atcddd/en. Accessed 10 July 2013 